\title{
On the optimal control of a two-queue polling model
}

\author{
F. Avram ${ }^{a}$, A. Gómez-Corral ${ }^{b, *}$ \\ a Department de Mathematiques, Université de Pau et des Pays de l'Adour, Avenue de l'université, 64000-Pau, \\ France \\ ${ }^{b}$ Department of Statistics and Operations Research I, Faculty of Mathematics, Complutense University of \\ Madrid, 28040-Madrid, Spain
}

\begin{abstract}
This paper deals with two $M / M / 1$ queues served by a single server with threshold switching. Our main goal is to solve the Poisson equation and, as a result, give expressions for the long-run expected average cost of holding units and switching actions of the server, and the bias vector.
\end{abstract}

Keywords: Poisson equation; Polling system; Priority queue; Threshold policy.

\section{Introduction}

An important problem that must be addressed in ATM networks is the provision of different levels of service to different types of traffic (e.g., voice and data). A method for achieving such a discrimination relies on the use of priority queues. Clearly, pure priority policies result in heavily penalized lower-priority streams. Hence, it is advisable to devise policies offering acceptable performance for lower-priority units, while still meeting the service requirements of higher-priority units. In order to overcome this shortcoming, several priority queues have been recently proposed and studied in the literature; see e.g. $[1,4,7,11,15]$.

Here our interest is in the queueing model introduced by Boxma, Koole and Mitrani [4], who study the influence of thresholds on polling models. Specifically, they consider a model of two $M / M / 1$ queues which are served by a single server (see Figure 1). There are two classes of units, called type1 and type- 2 units, which arrive according to two independent Poisson streams with rates $\lambda_{1}$ and $\lambda_{2}$, respectively. Type- 1 and type- 2 units are accommo- dated into two queues with infinite capacities and service scheduling depending on the length of the type-1 queue. To be concrete, the server switches from type- 2 queue to type-1 queue when either the latter reaches size $T$ or the former becomes empty, provided that type- 1 queue is not empty. The server switches from type-1 queue to type-2 queue only when the former becomes empty and the latter is not empty. Service process at type-1 queue is always exhaustive. The service time of a type- $k$ unit has exponential distribution with mean $1 / \mu_{k}$, for $k \in\{1,2\}$. Preemptions and server reallocations do not result in any delay. Assume that the arrival and service time processes are mutually independent processes. For later use, we let $\lambda_{k} / \mu_{k}$ be $\rho_{k}$, for $k \in\{1,2\}$.

Boxma, Koole and Mitrani [4] determine the joint queue length distribution in the above polling model, even when switching actions are only permitted at service completions, both using analytic techniques and using the power series algorithm. In this paper we focus on the optimal switching policy for such a model with holding and switching costs.

\footnotetext{
*Corresponding author. Phone: +34 913944473. Fax: +34 913944606. E-mail addresses: florin.avram@univ-pau.fr (F. Avram); antonio_gomez@mat.ucm.es (A. Gómez-Corral).
} 


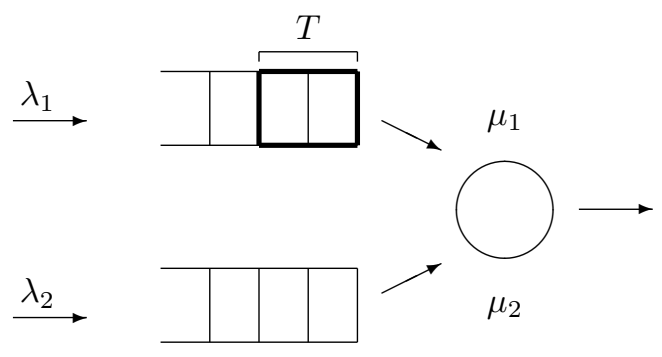

Figure 1. Polling model with threshold policy

If $c_{k}$ denotes the cost per unit time of keeping a type- $k$ unit in the system, then it has been shown that the well-known $c \mu$-rule [5] minimizes the total average cost; see also $[10,23]$ for a brief review of the subject. The $c \mu$-rule can be thought of as a static decision rule giving always higher priority to units with larger values of $c_{k} \mu_{k}$, so that the prioritization of units does not depend on the arrival rates $\lambda_{k}$. Nevertheless, if switching costs are positive, then the $c \mu$-rule is no longer optimal in general. Note that, in a heuristical manner, the $c \mu$-rule does not seem to govern the dynamics of our control problem because such a policy gives rise to a considerable amount of undesirable switchings of the server back and forth between the lines $\left\{\left(X_{1}(t), X_{2}(t)\right)=(0, n): n \geq 0\right\}$ and $\left\{\left(X_{1}(t), X_{2}(t)\right)=(1, n): n \geq 0\right\}$, where $X_{k}(t)$ is the number of type- $k$ units at time $t$, for $k \in\{1,2\}$.

Koole [12] has investigated this control problem extensively and found numerically that the optimal policy could be pretty complicated in the general case, where costs to be minimized consist of both holding and switching costs. For the special case $\mu_{k} \equiv \mu$ and $c_{k} \equiv c$, Hofri and Ross [9] (see also [16]) showed that the optimal policy serves each queue exhaustively, and conjectured that a threshold policy should be optimal for the general case. The main contribution of [12] is to illustrate the fact that the optimal policy cannot easily be characterized and can only be determined using an iterative procedure.

Our main objective is to investigate the solution of the Poisson equation - also known as dynamic programming optimality equation - and the bias vector. This result is relevant to a further onestep policy improvement [17] for deriving approximations to complex systems. Although a discussion of the subject is outside the scope of this work, we stress that the most remarkable in applying onestep policy improvement is that for specific policies the average cost and the value function of complex systems can be explicitly derived and, as a result, one-step policy iteration may result in an acceptable approximation to the optimal policy of the system. For applications of one-step policy improvement to a variety of cases, the reader is referred to $[2,8,13,14]$.

The paper is organized as follows. Some preliminary results on our control problem and the stationary distribution of the polling model under study [4] are presented briefly in Section 2 to make subsequent reading self contained. By studying the Poisson equation, in Section 3, we give expressions for the long-run expected average cost and the bias vector. Interestingly, our exact solution makes appear two fundamental quantities, $K$ and $f(T)$, which are very close to Reiman and Wein's heuristically derived diffusion solution [20]. Some further comments in Section 4 link such a diffusion control problem [20] to an optimal choice of the threshold policy.

\section{Preliminaries}

The polling model in [4] can be studied as a continuous-time Markov chain (CTMC) $\mathcal{X}=$ $\left\{\left(X_{1}(t), X_{2}(t), Y(t)\right): t \geq 0\right\}$, where $Y(t)$ equals $k$ if the server is at type- $k$ queue at time $t$, for $k \in\{1,2\}$. Clearly, $\mathcal{X}$ is an irreducible CTMC on the state space $\mathcal{S}=\{(0,0, k): k=1,2\} \cup\{(m, n, 1): m \geq$ $1, n \geq 0\} \cup\{(m, n, 2): 0 \leq m \leq T-1, n \geq 1\}$.

From now on we assume that the traffic load $\rho \equiv \rho_{1}+\rho_{2}$ is less than one, so the ergodicity condition is satisfied.

\subsection{Statement of the problem}

There are holding costs $c_{k}$, for $k \in\{1,2\}$, for each time unit a type- $k$ unit spends in a queue, and switching costs $s_{j}$, for $j \in\{1, \ldots, 5\}$, when the server switches from one class to the other; to keep the description simple, transitions among states of $\mathcal{X}$ associated with switching actions of the server are summarized in Table 1.

\begin{tabular}{lc}
\hline Transition & Cost \\
\hline$(1, n, 1) \rightarrow(0, n, 2), n \geq 1$ & $s_{1}$ \\
$(T-1, n, 2) \rightarrow(T, n, 1), n \geq 1$ & $s_{2}$ \\
$(0,0,1) \rightarrow(0,1,2)$ & $s_{3}$ \\
$(0,0,2) \rightarrow(1,0,1)$ & $s_{4}$ \\
$(m, 1,2) \rightarrow(m, 0,1), 1 \leq m \leq T-1$ & $s_{5}$ \\
\hline
\end{tabular}

Table 1. Switching costs 
Several optimality criteria are usually proposed to analyze CTMCs; see e.g. [19]. One of them is the average cost criterion, in which the limit of the expected average cost over finite time intervals is minimized in a set of predetermined policies. In our control problem, such an expected average cost, starting in state $(m, n, k)$ at time 0 , of the policy with threshold $T$, denoted by $\gamma_{(m, n, k)}(T)$, is of the form

$\lim _{t \rightarrow \infty} \frac{1}{t} E_{(m, n, k)}\left[\int_{0}^{t} \sum_{k=1}^{2} c_{k} X_{k}(u) d u+\sum_{j=1}^{5} s_{j} N_{j}(t)\right]$,

where $N_{j}(t)$ is the total number of passages in $(0, t]$ when our CTMC switches from one class to the other according to the switching cost $s_{j}$, and $E_{(m, n, k)}$ represents the conditional expectation of a random variable, given that $\left(X_{1}(0), X_{2}(0), Y(0)\right)=$ $(m, n, k)$. Note that the existence of a single ergodic class in $\mathcal{X}$ implies that $\gamma_{(m, n, k)}(T)$ is a constant function of $(m, n, k) \in \mathcal{S}$, which is denoted from now on by $\gamma(T)$.

From the general theory for optimal control of CTMCs [19, Chapter 11], the constant $\gamma(T)$ can be determined by solving the Poisson equation given by

$$
\gamma(T)=c(a)+\sum_{b \in \mathcal{S}} q_{a b} \omega(b), \quad a \in \mathcal{S}
$$

where the function $c: \mathcal{S} \rightarrow \mathbb{R}$ is the cost rate and $q_{a b}$, for $a, b \in \mathcal{S}$, are the infinitesimal transition rates of $\mathcal{X}$. In what follows, we solve the above Poisson equation (2) and consequently find the constant $\gamma(T)$ defined by (1) and a function $\omega: \mathcal{S} \rightarrow \mathbb{R}$, usually referred to as bias vector. For each state $(m, n, k) \in \mathcal{S}$, the bias $\omega(m, n, k)$ amounts to the total difference of costs starting from $(m, n, k)$ instead of a fixed reference state $\left(m_{0}, n_{0}, k_{0}\right)$.

Uniqueness of the pair $(\gamma(T), \omega)$, as a solution to $(2)$, is not guaranteed when dealing with denumerable CTMCs with unbounded cost functions, which is the case here. Without going into details, Bhulai and Speiksma [3] and Prieto-Rumeau and Hernández-Lerma [18] have shown recently that the solution $(\gamma(T), \omega)$ to the Poisson equation exists and is unique in the Banach space $\mathbb{B}_{v}(\mathcal{S})$ of bounded real-valued functions $u$ on $\mathcal{S}$ with a finite $v$-norm (or weighted supremum norm) $\|\cdot\|_{v}$, which is defined as

$$
\|u\|_{v}=\sup _{a \in \mathcal{S}} \frac{|u(a)|}{v(a)}
$$

where $v \geq 1$ is a suitably chosen function on $\mathcal{S}$ (see [3, Section 2] and [18, Assumptions A-C]). We stress here that the constant $\gamma(T)$ of such a unique solution corresponds to the expected average cost defined by (1), and that the bias vector $\omega$ is unique up to an additive constant. For the preemptive static priority studied by Groenevelt, Koole and Nain [8] (i.e., $T=1$ ), the reader is directed to $[3$, Section 5] for a full proof of the uniqueness of the pair $(\gamma(1), \omega)$ within a certain normed space. Due to the nature of our solution in Theorem 1, we conjecture the uniqueness of $(\gamma(T), \omega)$, but the extension of Bhulai and Spieksma's arguments [3] to the case $T>1$ is not immediate.

To determine the pair $(\gamma(T), \omega)$, we first choose $(0,0,2)$ as reference state to fix $\omega(0,0,2)=0$. We then extend the definition of the bias vector to the set $\mathcal{S}^{\prime}=\{(m, n, 1): m \geq 0, n \geq 0\} \cup\{(m, n, 2)$ : $0 \leq m \leq T, n \geq 0\}$ by setting

$$
\begin{aligned}
\omega(0, n, 1) & =\omega(0, n, 2)+s_{1}, \quad n \geq 1 \\
\omega(T, n, 2) & =\omega(T, n, 1)+s_{2}, \quad n \geq 1 \\
\omega(m, 0,2) & =\omega(m, 0,1)+s_{5}, 1 \leq m \leq T-1
\end{aligned}
$$

which correspond (see Table 1) to server switchings immediately after the service completion of a type1 unit, the arrival of a type- 1 unit and the service completion of a type-2 unit, respectively.

For states in $\mathcal{S},(2)$ consists of the equalities

$$
\begin{aligned}
\gamma(T)= & \lambda_{1}(\omega(m+1, n, 1)-\omega(m, n, 1)) \\
& +\lambda_{2}(\omega(m, n+1,1)-\omega(m, n, 1)) \\
& +\mu_{1}(\omega(m-1, n, 1)-\omega(m, n, 1)) \\
& +c_{1} m+c_{2} n, \quad m \geq 2, n \geq 1 \\
\gamma(T)=\quad & \lambda_{1}(\omega(2, n, 1)-\omega(1, n, 1)) \\
& +\lambda_{2}(\omega(1, n+1,1)-\omega(1, n, 1)) \\
& +\mu_{1}\left(\omega(0, n, 2)-\omega(1, n, 1)+s_{1}\right) \\
& +c_{1}+c_{2} n, \quad n \geq 1 \\
\gamma(T)= & \lambda_{1}(\omega(m+1,0,1)-\omega(m, 0,1)) \\
& +\lambda_{2}(\omega(m, 1,1)-\omega(m, 0,1)) \\
& +\mu_{1}(\omega(m-1,0,1)-\omega(m, 0,1)) \\
& +c_{1} m, \quad m \geq 1, \\
& \lambda_{1}(\omega(1,0,1)-\omega(0,0,1)) \\
& +\lambda_{2}\left(\omega(0,1,2)-\omega(0,0,1)+s_{3}\right) \\
\gamma(T)= & \lambda_{1}\left(\omega(1,0,1)+s_{4}\right)+\lambda_{2} \omega(0,1,2)
\end{aligned}
$$




$$
\begin{aligned}
\gamma(T)= & \lambda_{1}(\omega(m+1, n, 2)-\omega(m, n, 2)) \\
& +\lambda_{2}(\omega(m, n+1,2)-\omega(m, n, 2)) \\
& +\mu_{2}(\omega(m, n-1,2)-\omega(m, n, 2)) \\
& +c_{1} m+c_{2} n, 0 \leq m \leq T-2, n \geq 2,(11) \\
\gamma(T)= & \lambda_{1}\left(\omega(T, n, 1)-\omega(T-1, n, 2)+s_{2}\right) \\
& +\lambda_{2}(\omega(T-1, n+1,2)-\omega(T-1, n, 2)) \\
& +\mu_{2}(\omega(T-1, n-1,2)-\omega(T-1, n, 2)) \\
& +c_{1}(T-1)+c_{2} n, \quad n \geq 2, \\
\gamma(T)= & \lambda_{1}(\omega(1,1,2)-\omega(0,1,2)) \\
& +\lambda_{2}(\omega(0,2,2)-\omega(0,1,2)) \\
& +\mu_{2}(\omega(0,0,2)-\omega(0,1,2))+c_{2}, \\
\gamma(T)= & \lambda_{1}(\omega(m+1,1,2)-\omega(m, 1,2)) \\
& +\lambda_{2}(\omega(m, 2,2)-\omega(m, 1,2)) \\
& +\mu_{2}\left(\omega(m, 0,1)-\omega(m, 1,2)+s_{5}\right) \\
& +c_{1} m+c_{2}, \quad 1 \leq m \leq T-2, \\
\gamma(T)= & \lambda_{1}\left(\omega(T, 1,1)-\omega(T-1,1,2)+s_{2}\right) \\
& +\lambda_{2}(\omega(T-1,2,2)-\omega(T-1,1,2)) \\
& +\mu_{2}\left(\omega(T-1,0,1)-\omega(T-1,1,2)+s_{5}\right) \\
& +c_{1}(T-1)+c_{2} .
\end{aligned}
$$

By (3)-(5), Equations (6)-(8) and (11)-(15) may further be simplified to yield

$$
\begin{aligned}
\gamma(T)= & \lambda_{1}(\omega(m+1, n, 1)-\omega(m, n, 1)) \\
& +\lambda_{2}(\omega(m, n+1,1)-\omega(m, n, 1)) \\
& +\mu_{1}(\omega(m-1, n, 1)-\omega(m, n, 1)) \\
& +c_{1} m+c_{2} n, \quad m \geq 1, n \geq 0 \\
\gamma(T)= & \lambda_{1}(\omega(m+1, n, 2)-\omega(m, n, 2)) \\
& +\lambda_{2}(\omega(m, n+1,2)-\omega(m, n, 2)) \\
& +\mu_{2}(\omega(m, n-1,2)-\omega(m, n, 2)) \\
& +c_{1} m+c_{2} n, 0 \leq m \leq T-1, n \geq 1 .(17)
\end{aligned}
$$

At this time, for ease of presentation, we digress to briefly recall the analytic solution for the stationary distribution of $\mathcal{X}$ given in [4, Section 2].

\subsection{Auxiliary results on the generat- ing function of the stationary dis- tribution}

This subsection is used to introduce notation and collect some results that will be used in Section 3 .

The stationary distribution of $\mathcal{X}$, say $\pi$, consists of the limiting probabilities

$$
r_{k}=\lim _{t \rightarrow \infty} P\left(X_{1}(t)=0, X_{2}(t)=0, Y(t)=k\right),
$$

$k \in\{1,2\}$,

$p_{m n}=\lim _{t \rightarrow \infty} P\left(X_{1}(t)=m, X_{2}(t)=n, Y(t)=1\right)$,

$m \geq 1, n \geq 0$,

$q_{m n}=\lim _{t \rightarrow \infty} P\left(X_{1}(t)=m, X_{2}(t)=n, Y(t)=2\right)$,

$$
0 \leq m \leq T-1, n \geq 1 \text {. }
$$

Using an analytic approach [4] based on generating functions and zeros of analytic functions allows us to derive expressions for $r_{k}$, for $k \in\{1,2\}$, $P(x, y)=\sum_{m=1}^{\infty} x^{m-1} \sum_{n=0}^{\infty} y^{n} p_{m n}$ and $Q_{m}(y)=$ $\sum_{n=1}^{\infty} y^{n-1} q_{m n}$, for $0 \leq m \leq T-1$. To do that, we need some notation:

$$
\begin{aligned}
K(x, y) & \equiv \lambda_{1}(1-x)+\lambda_{2}(1-y)+\mu_{1}\left(1-x^{-1}\right), \\
a(y) & \equiv \lambda_{1}+\lambda_{2}(1-y)+\mu_{2}\left(1-y^{-1}\right) .
\end{aligned}
$$

In short, the probabilities $r_{k}$ and the generating functions $P(x, y)$ and $Q_{m}(y)$ satisfy

$$
\begin{aligned}
& r_{1}=\frac{1}{\lambda_{1}+\lambda_{2}}\left(\lambda_{1}(1-\rho)+\mu_{2} \sum_{m=1}^{T-1} Q_{m}(0)\right), \\
& r_{2}=\frac{1}{\lambda_{1}+\lambda_{2}}\left(\lambda_{2}(1-\rho)-\mu_{2} \sum_{m=1}^{T-1} Q_{m}(0)\right),
\end{aligned}
$$

$$
\begin{aligned}
K(x, y) P(x, y)= & \lambda_{1}(1-\rho)+\lambda_{1} x^{T-1} y Q_{T-1}(y) \\
& -\mu_{1} x^{-1} P(0, y) \\
& +\mu_{2} \sum_{m=1}^{T-1} x^{m-1} Q_{m}(0), \\
Q_{m}(y)= & \left(\frac{\lambda_{1}}{a(y)}\right)^{m} Q_{0}(y) \\
& -\frac{\mu_{2}}{y a(y)} \sum_{l=1}^{m}\left(\frac{\lambda_{1}}{a(y)}\right)^{m-l} Q_{l}(0),
\end{aligned}
$$

for $1 \leq m \leq T-1$. To find an expression for $P(0, y)$, we note that, for every $y$ of the open unit disk $|y|<1$, the kernel $K(x, y)$ has a unique zero $x=\alpha(y)$ in $|x|<1$, which corresponds (see [21, page 34]) to the generating function of the number of arrivals to the type- 2 queue during a busy period (with length $\beta_{1}$ ) in the $M / M / 1$ queue with arrival rate $\lambda_{1}$ and service rate $\mu_{1}$; i.e., it is given by $\alpha(y)=E\left[\exp \left\{-\lambda_{2}(1-y) \beta_{1}\right\}\right]$ and satisfies

$\lambda_{1} \alpha^{2}(y)-\left(\lambda_{1}+\lambda_{2}(1-y)+\mu_{1}\right) \alpha(y)+\mu_{1}=0$,

in the open unit disk. Clearly, $P(x, y)$ is analytic in the poly-disk $|x| \leq 1,|y| \leq 1$, so that the righthand side of (20) must vanish for all zeros $x=\alpha(y)$ 
of the kernel in $|y| \leq 1$, from which it follows the equality

$$
\begin{aligned}
\mu_{1} P(0, y)= & \lambda_{1}\left((1-\rho) \alpha(y)+y \alpha^{T}(y) Q_{T-1}(y)\right) \\
& +\mu_{2} \sum_{m=1}^{T-1} \alpha^{m}(y) Q_{m}(0) .
\end{aligned}
$$

Hence, Equations (18)-(23) determine the solution once $Q_{0}(y)$ is computed and the $T-1$ unknown constants $Q_{m}(0)$, for $1 \leq m \leq T-1$, are evaluated. Additional equations for $Q_{0}(y)$ and these unknowns are obtained from

$$
\begin{aligned}
u(y) Q_{0}(y)= & (y a(y))^{T-1} \\
& \times\left(g(y)+\sum_{m=1}^{T-1} h_{m}(y) Q_{m}(0)\right),
\end{aligned}
$$

and by noting that the right-hand side of (24) must vanish whenever $u(y)$ vanishes in the open unit disk $|y|<1$, where

$$
\begin{aligned}
g(y) & =(1-\rho)\left(\lambda_{1}(\alpha(y)-1)+\lambda_{2}(y-1)\right), \\
u(y) & =(y a(y))^{T}\left(1-\left(\frac{\lambda_{1} y \alpha(y)}{y a(y)}\right)^{T}\right), \\
h_{m}(y) & =\mu_{2} \alpha^{m}(y)\left(1-\left(\frac{\lambda_{1} y \alpha(y)}{y a(y)}\right)^{T-m}\right),
\end{aligned}
$$

for $1 \leq m \leq T-1$. An appeal to Rouche's theorem allows us to assert that $u(y)$ has exactly $T-1$ zeros strictly in the open unit disk. Precisely, for $1 \leq m \leq T-1$, the equation $e_{m} y a(y)=\lambda_{1} y \alpha(y)$, where $e_{m}=\exp \{2 m i \pi / T\}$ is the $m$ th root of unity of order $T$, has a single root $y_{m}$ in the open unit disk. Furthermore, $y_{m}$ satisfies the equation

$\lambda_{2} y^{2}-\left(\lambda_{1}\left(1-\frac{\alpha(y)}{e_{m}}\right)+\lambda_{2}+\mu_{2}\right) y+\mu_{2}=0$,

from which $y_{m}$ can be written in terms of the length $\beta_{2}$ of a busy period in the type- 2 queue; i.e., $y_{m}=E\left[\exp \left\{-\lambda_{1}\left(1-\alpha\left(y_{m}\right) / e_{m}\right) \beta_{2}\right\}\right]$. Then, by substituting the $T-1$ roots $y_{m}$ into Equation (24), the $T-1$ unknowns $Q_{m}(0)$ can be evaluated as the solution of

$$
\sum_{l=1}^{T-1} h_{l}\left(y_{m}\right) Q_{l}(0)=-g\left(y_{m}\right),
$$

for $1 \leq m \leq T-1$. In matrix form, the solution to (25) has the form

$$
\mathbf{Q}(0)=-\mathbf{H}^{-1} \mathbf{g},
$$

where $\mathbf{Q}(0)$ and $\mathbf{g}$ are column vectors of order $T-1$ with $m$ th entries $Q_{m}(0)$ and $g\left(y_{m}\right)$, respectively, and $\mathbf{H}$ is the square matrix of order $T-1$ with $(m, l)$ th entry

$$
h_{l}\left(y_{m}\right)=\mu_{2}\left(\alpha^{l}\left(y_{m}\right)-\left(\frac{a\left(y_{m}\right)}{\lambda_{1}}\right)^{l}\right),
$$

whence $\mathbf{H}$ is a difference of Vandermonde matrices.

\section{The Poisson equation and its solution}

Next we focus on the solution to the set of equivalent equations (3)-(5), (9), (10), (16) and (17). We will use notation and results in Subsection 2.2 as part of our analysis here. For example, the solution in Theorem 1 uses the matrix $\mathbf{H}$ and the vector $\mathbf{g}$ of (26), and Remark 1 connects the stationary distribution in (18)-(25) to our solution in Theorem 1.

Our first step is to define the constants

$$
\begin{aligned}
K & =\frac{c_{1} \mu_{1}-c_{2} \mu_{2}}{2 \lambda_{1} \mu_{1}\left(1-\rho_{1}\right)} \\
f(T) & =\frac{s_{1}+s_{2}}{T}+K T, \\
b_{0}= & \frac{1}{2 \lambda_{1}}\left(\frac{\mu_{2}\left(1-\rho_{2}\right) c_{2}}{\mu_{1}(1-\rho)}-c_{1}\right), \\
b_{1}= & \frac{c_{1}+\lambda_{2} b_{3}}{2 \mu_{1}\left(1-\rho_{1}\right)} \\
b_{2}= & \frac{c_{2}}{2 \mu_{2}(1-\rho)}, \\
b_{3}= & \frac{c_{2}}{\mu_{1}(1-\rho)}, \\
b_{0}^{\prime}= & K+f(T)+b_{1}^{\prime}, \\
b_{1}^{\prime}= & \rho_{1}\left((z-1) s_{1}+\frac{\left(\lambda_{1}(z-1)-\lambda_{2}\right) s_{4}}{\lambda_{1}+\lambda_{2}}\right. \\
& \left.-\frac{\lambda_{2} z s_{3}}{\lambda_{1}+\lambda_{2}}\right)+\sum_{l=1}^{T-1} z_{l} \frac{1-\alpha_{l}}{\alpha_{l}}, \\
b_{2}^{\prime}= & \frac{\mu_{1}}{\mu_{2}}\left(\rho_{1}(f(T)-K)+b_{1}^{\prime}\right), \\
b_{3}^{\prime}= & s_{1}+\frac{\lambda_{1} s_{4}-\lambda_{2} s_{3}}{\lambda_{1}+\lambda_{2}},
\end{aligned}
$$

where $\alpha_{l} \equiv \alpha\left(y_{l}\right)$ and $z$ is the root in $|z|<1$ of $\lambda_{1} z^{2}-\left(\lambda_{1}+\lambda_{2}+\mu_{1}\right) z+\mu_{1}=0$. 
Consider now the vector $\mathbf{z}=\left[z_{1}, \ldots, z_{T-1}\right]$ such that $\mu_{2}^{-1} \mathbf{z}=f(T) \mathbf{z}_{1} \mathbf{H}^{-1}+\mathbf{z}_{2} \mathbf{H}^{-1}$, where $\mathbf{z}_{1}$ and $\mathbf{z}_{2}$ are respectively the $(T-1)$-dimensional row vectors with $l$ th entries given by $l$ and $-K l^{2}+$ $b_{3}^{\prime} z^{l}-\left(s_{1}+s_{5}\right)$ for $1 \leq l \leq T-1$. We denote $\sum_{l=1}^{T-1} z_{l}\left(\alpha_{l}^{m} y_{l}^{n}-1\right)$ and $\sum_{l=1}^{T-1} z_{l}\left(a_{l}^{m} y_{l}^{n}-1\right)$ by $Z(m, n, 1)$ and $Z(m, n, 2)$, respectively, with $a_{l} \equiv$ $\alpha_{l} / e_{l}$.

The following result lists properties of $Z(m, n, k)$, for $k \in\{1,2\}$. Its proof is straightforward from Subsection 2.2 and we thus omit it.

Lemma 1. (i) $Z(0,0,1)=Z(0,0,2)=0$, $Z(0, n, 1)=Z(0, n, 2)$, for $n \geq 1$, and $Z(T, n, 1)=$ $Z(T, n, 2)$, for $n \geq 1$.

(ii) The functions $Z(m, n, 1)$, for $m \geq 1$ and $n \geq 0$, satisfy the set of equations

$$
\begin{aligned}
& \lambda_{1}(Z(m+1, n, 1)-Z(m, n, 1)) \\
& \quad+\lambda_{2}(Z(m, n+1,1)-Z(m, n, 1)) \\
& \quad+\mu_{1}(Z(m-1, n, 1)-Z(m, n, 1))=0 .
\end{aligned}
$$

(iii) The functions $Z(m, n, 2)$, for $0 \leq m \leq T-1$ and $n \geq 1$, satisfy the set of equations

$$
\begin{aligned}
& \lambda_{1}(Z(m+1, n, 2)-Z(m, n, 2)) \\
& \quad+\lambda_{2}(Z(m, n+1,2)-Z(m, n, 2)) \\
& \quad+\mu_{2}(Z(m, n-1,2)-Z(m, n, 2))=0 .
\end{aligned}
$$

We are now in a position to address the computation of the pair $(\gamma(T), \omega)$.

Theorem 1. The bias vector $\omega(m, n, k)$ of our polling model is given by Equations (3)-(5) and

$$
\begin{aligned}
\omega(m, n, 1)= & q_{1}(m)+q_{2}(n)+Z(m, n, 1) \\
& +b_{3} m n+s_{1}, \quad m \geq 0, n \geq 1, \\
\omega(m, 0,1)= & q_{1}(m)+Z(m, 0,1) \\
& +b_{3}^{\prime}\left(1-z^{m}\right)+\omega(0,0,1), \quad m \geq 1, \\
\omega(m, n, 2)= & q_{0}(m)+q_{2}(n)+Z(m, n, 2), \quad(31) \\
& +b_{3} m n, \quad 0 \leq m \leq T, n \geq 1,
\end{aligned}
$$

where $q_{k}(m)=b_{k}\left(m^{2}+m\right)+b_{k}^{\prime} m$, for $k \in\{0,1\}$, $q_{2}(n)=b_{2}\left(n^{2}+n\right)+b_{2}^{\prime} n$, and $\omega(0,0,1)=\left(\lambda_{2} s_{3}-\right.$ $\left.\lambda_{1} s_{4}\right) /\left(\lambda_{1}+\lambda_{2}\right)$. Furthermore, $\gamma(T)$ has the form

$$
\gamma(T)=\gamma_{0}+\gamma_{1}(T),
$$

where $\gamma_{0}$ and $\gamma_{1}(T)$ are given by

$$
\gamma_{0}=2 \sum_{k=1}^{2} \lambda_{k} b_{k}-\lambda_{1} \rho_{2} K
$$

$$
\begin{aligned}
& +\lambda_{1}(1-\rho)\left((1-z) s_{1}+\frac{\lambda_{2} z}{\lambda_{1}+\lambda_{2}} s_{3}\right. \\
& \left.+\frac{\lambda_{1}(1-z)+\lambda_{2}}{\lambda_{1}+\lambda_{2}} s_{4}\right) \\
\gamma_{1}(T)= & \left(\lambda_{1} \rho_{2}+\mu_{2} \mathbf{Z}_{1} \mathbf{H}^{-1} \mathbf{g}\right) f(T)+\mu_{2} \mathbf{z}_{2} \mathbf{H}^{-1} \mathbf{g} .
\end{aligned}
$$

Proof. The proof consists in checking that the pair $(\gamma(T), \omega)$ defined in (3)-(5) and (29)-(32) satisfies the set of equations (9), (10), (16) and (17).

We begin with (10). Substituting $\gamma(T)$, $\omega(1,0,1)$ and $\omega(0,1,2)$ by the expressions given in (30)-(32) yields the value of $b_{3}^{\prime}$ we give as its definition.

We now consider Equation (9). By using expressions for $\omega(1,0,1), \omega(0,1,2)$ and $\gamma(T)$ given in (30)-(32) we easily obtain

$$
\lambda_{2} \omega(0,0,1)=\lambda_{1}\left(b_{3}^{\prime}-\left(s_{1}+s_{4}\right)\right)+\lambda_{2} s_{3},
$$

which allows us to derive the value of $\omega(0,0,1)$ by substitution of the constant $b_{3}^{\prime}$.

To check the validity of (3), we observe that (3) is equivalent to $Z(0, n, 1)=Z(0, n, 2)$, for $n \geq 1$, which is indeed correct from Lemma 1.

By introducing (29) and (31) into (4), it is easily seen that (4) reduces to $q_{0}(T)-q_{1}(T)=s_{1}+s_{2}$, since $Z(T, n, 1)=Z(T, n, 2)$, for $n \geq 1$, by Lemma 1. Then this identity is true from the definition of the constants $b_{k}$ and $b_{k}^{\prime}$, for $k \in\{0,1\}$.

If we replace $\omega(m, 0,1)$ and $\omega(m, 0,2)$ in (5) by their expressions given in (30) and (31), respectively, then we get

$$
\begin{aligned}
Z(m, 0,1)-Z(m, 0,2)= & q_{0}(m)-q_{1}(m)+b_{3}^{\prime} z^{m} \\
& -\left(s_{1}+s_{5}\right)
\end{aligned}
$$

for $1 \leq m \leq T-1$, where we may write down $q_{0}(m)-q_{1}(m)=(f(T)-K m) m$. Using now the definition of the values $z_{l}$, it is straightforward to check that indeed the above identity holds true since $Z(m, 0,1)-Z(m, 0,2)=\sum_{l=1}^{T-1} z_{l} a_{l}^{m}\left(e_{l}^{m}-1\right)$, for $1 \leq m \leq T-1$. Note that the right-hand side of the above equation allows us to notice that (33) corresponds to the $m$ th equation of the matrix equation $\mathbf{z H}=\mu_{2}\left(f(T) \mathbf{z}_{1}+\mathbf{z}_{2}\right)$, which completes the proof of (5).

To check the validity of (16), we first study the case $m \geq 1$ and $n \geq 1$. By replacing $\omega(m+1, n, 1)$, $\omega(m, n+1,1), \omega(m-1, n, 1)$ and $\omega(m, n, 1)$ in $(16)$ by their expressions given in (29), and dropping 
terms that appear on both sides of the resulting equation, we have

$$
\begin{aligned}
\gamma(T)= & \lambda_{1}\left(2 b_{1}(m+1)+b_{1}^{\prime}+b_{3} n\right) \\
& +\lambda_{2}\left(2 b_{2}(n+1)+b_{2}^{\prime}+b_{3} m\right) \\
& -\mu_{1}\left(2 b_{1} m+b_{1}^{\prime}+b_{3} n\right),
\end{aligned}
$$

for $m \geq 1$ and $n \geq 1$, by (27). By equating coefficients of $m$ and $n$, we may write down

$$
\gamma(T)+\mu_{1} b_{1}^{\prime}=\lambda_{1}\left(2 b_{1}+b_{1}^{\prime}\right)+\lambda_{2}\left(2 b_{2}+b_{2}^{\prime}\right),
$$

which holds true by using (32) and the definition of $b_{k}$ and $b_{k}^{\prime}$, for $k \in\{1,2\}$. In the case $m \geq 1$ and $n=0$, a direct substitution of the expressions for $\omega(m+1,0,1), \omega(m, 1,1), \omega(m-1,0,1)$ and $\omega(m, 0,1)$ given in (29) and (30) yields

$$
\begin{aligned}
\gamma(T)= & \lambda_{1}\left(2 b_{1}(m+1)+b_{1}^{\prime}\right) \\
& +\lambda_{2}\left(b_{3} m+2 b_{2}+b_{2}^{\prime}+s_{1}-\omega(0,0,1)\right) \\
& -\mu_{1}\left(2 b_{1} m+b_{1}^{\prime}\right)+c_{1} m \\
& +\left(\lambda_{1}(1-z)+\mu_{1}\left(1-z^{-1}\right)\right) b_{3}^{\prime} z^{m} \\
& +\lambda_{2} b_{3}^{\prime}\left(z^{m}-1\right),
\end{aligned}
$$

by (27). Then by taking $z^{m}=\sum_{l=0}^{\infty}(m \log z)^{l} / l$ ! into account and by equating coefficients of $m$, we get

$$
\begin{aligned}
\gamma(T)= & \lambda_{1}\left(2 b_{1}+b_{1}^{\prime}\right)-\mu_{1} b_{1}^{\prime} \\
& +\lambda_{2}\left(2 b_{2}+b_{2}^{\prime}-b_{3}^{\prime}+s_{1}-\omega(0,0,1)\right),
\end{aligned}
$$

since $\lambda_{1}(1-z)+\mu_{1}\left(1-z^{-1}\right)=-\lambda_{2}$. The above equality then follows directly from (32) and the definition of $b_{k}$ and $b_{k}^{\prime}$, for $k \in\{1,2\}$, and $b_{3}^{\prime}$.

To conclude, we consider (17). From (28) and (31), we readily note that (17) amounts to

$$
\begin{aligned}
\gamma(T) & =\lambda_{1}\left(2 b_{0}+b_{0}^{\prime}\right)+\lambda_{2}\left(2 b_{2}+b_{2}^{\prime}\right)-\mu_{2} b_{2}^{\prime} \\
0 & =2 \lambda_{1} b_{0}+\left(\lambda_{2}-\mu_{2}\right) b_{3}+c_{1} \\
0 & =\lambda_{1} b_{3}+2\left(\lambda_{2}-\mu_{2}\right) b_{2}+c_{2},
\end{aligned}
$$

so the validity of (17) reduces to a direct substitution of the constants $b_{k}$ and $b_{k}^{\prime}$, for $k \in\{0,2\}, b_{3}$ and the expression in (32) for $\gamma(T)$.

It should be noted that the terms $b_{3}^{\prime}\left(1-z^{m}\right)$ and $Z(m, n, k)$, for $k \in\{1,2\}$, in Theorem 1 reflect the non-linear/quadratic dependence of the bias vector on the pair $(m, n)$. Clearly, $b_{3}^{\prime}\left(1-z^{m}\right)$ does not depend on $T$ (indeed, it appears in Theorem 3.1 of [8]). However, the functions $Z(m, n, k)$, for $k \in\{1,2\}$, are characteristic features of each threshold $T$ and are not merely the result of formal manipulations. To justify the form of $Z(m, n, k)$, we may emphasize the similarity between statements (ii) and (iii) in Lemma 1 and the right-hand sides of Equations (16) and (17). We also favor the following remark, which holds by appealing to the general theory for CTMCs [6].

Remark 1. In terms of the stationary distribution $\pi$ of $\mathcal{X}$, the constant $\gamma(T)$ may be written as

$$
\begin{aligned}
\gamma(T)= & c_{1} E\left[X_{1}\right]+c_{2} E\left[X_{2}\right] \\
& +s_{1} \mu_{1} \pi\{(1, n, 1): n \geq 1\} \\
& +s_{2} \lambda_{1} \pi\{(T-1, n, 2): n \geq 1\} \\
& +s_{3} \lambda_{2} \pi\{(0,0,1)\}+s_{4} \lambda_{1} \pi\{(0,0,2)\} \\
& +s_{5} \mu_{2} \pi\{(m, 1,2): 1 \leq m \leq T-1\},
\end{aligned}
$$

(see $[6$, Section 8.5$]$ ) where $E\left[X_{k}\right]$ is the stationary expectation of the type- $k$ queue length, for $k \in\{1,2\}$. From (18)-(25), straightforward algebra yields the following mean values and probabilities:

$$
\begin{aligned}
& E\left[X_{1}\right]= \frac{1}{1-\rho_{1}}\left(\rho_{1}+\frac{\rho_{2}(T-1)}{2}\right) \\
&-\frac{\mu_{2}}{2 \lambda_{1}\left(1-\rho_{1}\right)} \sum_{m=1}^{T-1}(T-m) m Q_{m}(0), \\
& E\left[X_{2}\right]= \frac{1}{1-\rho}\left(\frac{\rho_{1} \lambda_{2}}{\mu_{1}\left(1-\rho_{1}\right)}+\rho_{2}\right)-\frac{\lambda_{2}(T-1)}{2 \mu_{1}\left(1-\rho_{1}\right)} \\
&+\frac{\mu_{2}^{2}}{2 \lambda_{1} \mu_{1}\left(1-\rho_{1}\right)} \sum_{m=1}^{T-1}(T-m) m Q_{m}(0), \\
& \pi\{(1, n, 1): n \geq 1\}=\frac{\rho_{1} \rho_{2}}{T}-\frac{\mu_{2}}{T \mu_{1}} \sum_{m=1}^{T-1} m Q_{m}(0), \\
& \pi\{(T-1, n, 2): n \geq 1\}=\frac{\rho_{2}}{T}-\frac{\mu_{2}}{T \lambda_{1}} \sum_{m=1}^{T-1} m Q_{m}(0), \\
& \pi\{(m, 1,2): 1 \leq m \leq T-1\}=\sum_{m=1}^{T-1} Q_{m}(0) .
\end{aligned}
$$

Then it can be readily seen that the right-hand sides of (32) and (34) coincide by equating coefficients of $c_{k}$, for $k \in\{1,2\}$, and $s_{j}$, for $1 \leq j \leq 5$. To check this fact, the reader should consider the equalities

$\mu_{2} \sum_{m=1}^{T-1} z^{m} Q_{m}(0)=\lambda_{1}(1-\rho)(1-z)+\mu_{2} \sum_{m=1}^{T-1} Q_{m}(0)$ 


$$
\begin{aligned}
\sum_{l=1}^{T-1} z_{l} g\left(y_{l}\right)+ & \lambda_{1}(1-\rho)(1-z) b_{3}^{\prime} \\
= & \mu_{2}\left(\frac{s_{3} \lambda_{2}-s_{4} \lambda_{1}}{\lambda_{1}+\lambda_{2}}+s_{5}\right) \sum_{m=1}^{T-1} Q_{m}(0) \\
& +\mu_{2} \sum_{m=1}^{T-1}(K m-f(T)) m Q_{m}(0)
\end{aligned}
$$

which are obtained from (20), (26) and the identity $\mu_{1}(1-\rho)\left(\alpha\left(y_{m}\right)-1\right) / \alpha\left(y_{m}\right)=g\left(y_{m}\right)$.

Furthermore, Theorem 1 has the following immediate consequence.

Remark 2. The solution to the preemptive static priority given by Groenevelt, Koole and Nain [8] can be derived from Theorem 1 once we take $(0,0,1)$ as reference state; i.e., the bias vector in [8], denoted by $h(m, n, k)$, is obtained by letting $h(m, n, k)=\omega(m, n, k)-\omega(0,0,1)$ in the particular case of switching costs $s_{3}=s_{1}, s_{4}=s_{2}$ and $s_{5}=s_{2}$ as the reader may easily verify.

For the preemptive static priority [8], we now interpret some terms of the solution. Our interpretations are based on the simpler "fluid problem" in which the process is replaced by its expectation, and which is known to provide the dominant terms when $x_{1}=m$ and $x_{2}=n$ converge to infinity.

Remark 3. For the case $T=1$, the process in the fluid problem follows the deterministic field $x_{1}^{\prime}=\lambda_{1}-\mu_{1}, x_{2}^{\prime}=\lambda_{2}$ while the server works at type- 1 queue, and the field $x_{1}^{\prime}=\lambda_{1}, x_{2}^{\prime}=\lambda_{2}-\mu_{2}$ while the server works at type- 2 queue. After first reaching the $x_{2}$ axis, the process viewed from far away evolves towards the origin spending some proportion of time $1-p_{1}$ exactly on the axis using the second deterministic field, and a proportion $p_{1}$ of the time using the first deterministic field. Since the resulting $x_{1}$ velocity $x_{1}^{\prime}=\left(1-p_{1}\right) \lambda_{1}+p_{1}\left(\lambda_{1}-\mu_{1}\right)=\lambda_{1}-p_{1} \mu_{1}$ must be zero, it follows that $p_{1}=\rho_{1}$. As a result, the resulting $x_{2}$ velocity is $x_{2}^{\prime}=\lambda_{2}-\mu_{2}\left(1-\rho_{1}\right)=\lambda_{2}-\tilde{\mu}_{2}$, where $\tilde{\mu}_{2}=\mu_{2}\left(1-\rho_{1}\right)$ is an adjusted service rate, which is due to the server working at type- 2 queue only part of the time. We will call the "combined" deterministic field $x_{1}^{\prime}=0, x_{2}^{\prime}=\lambda_{2}-\tilde{\mu}_{2}$ the "boundary deterministic" field.

Consider now the fluid "emptying path" until the origin starting at $\left(x_{1}, x_{2}\right)$ with the server work- ing at type- 1 queue initially. This will use first the first deterministic field, until the point $x_{1}=0$, $\tilde{x}_{2}=x_{2}+\lambda_{2} x_{1} /\left(\mu_{1}-\lambda_{1}\right)$, and after that it will use the boundary deterministic field. The resulting holding costs for the emptying of the first/second class are respectively $c_{1}$ times the area of a triangle with height $x_{1}$ and basis $T_{1}=x_{1} /\left(\mu_{1}-\lambda_{1}\right)$ and $c_{2}$ times the sum of the area of a trapezoid with heights $x_{2}, \tilde{x}_{2}$ and basis $T_{1}$ with that of a triangle with height $\tilde{x}_{2}$ and basis $T_{2}=x_{2} /\left(\tilde{\mu}_{2}-\lambda_{2}\right)$. Performing this computation we find the quadratic $q\left(x_{1}, x_{2}\right)=\sum_{k=1}^{2} b_{k} x_{k}^{2}+b_{3} x_{1} x_{2}$, from which it is easily checked in our problem that, for large values $(m, n)$, the fluid terms (i.e., associated with coefficients $b_{1}, b_{2}$ and $b_{3}$ ) will dominate asymptotically the other terms in the bias vector $\omega(m, n, 1)$, and also in $\omega(m, n, 2)$.

Remark 3 enriches those comments of [8] for the case $T=1$, which were made mainly in terms of busy periods of single server queues. Owing to the sophisticated expression for $(\gamma(T), \omega)$ in $(29)-(32)$, further work is necessary to interpret the solution in the general case $T>1$.

\section{Comments}

Theorem 1 can be viewed as the key step of a further one-step policy improvement. As initial policy we can take the $c \mu$-rule and, in the optimization step, we can use the expressions in Theorem 1 for a particular threshold $T^{*}$. A reasonable choice of $T^{*}$ might correspond to the threshold policy minimizing, in the set of threshold policies, the long-run expected average cost of holding units and switching actions of the server; note that the set of threshold policies includes the well studied preemptive static priority, obtained with $T=1$ (see [8]), and exhaustive service, obtained with $T=\infty$ (see [22]). To that end, we stress that $\gamma(T)$ depends on $T$ through the function $\tilde{\gamma}_{1}(T) \equiv \gamma_{1}(T)+\lambda_{1}(1-\rho)(1-z) b_{3}^{\prime}$ which is given by

$$
\begin{aligned}
& \tilde{\gamma}_{1}(T)=\left(\lambda_{1} \rho_{2}-\mu_{2} \sum_{m=1}^{T-1} m Q_{m}(0)\right) f(T) \\
& +\mu_{2} \sum_{m=1}^{T-1}\left(K m^{2}+\frac{s_{3} \lambda_{2}-s_{4} \lambda_{1}}{\lambda_{1}+\lambda_{2}}+s_{5}\right) Q_{m}(0),
\end{aligned}
$$

whence it might be more beneficial the use of a numerical approach instead of attempting to compute 
analytically the value of $T^{*}$ minimizing the above expression for $\tilde{\gamma}_{1}(\cdot)$.

In an attempt to derive an analytical expression for $T^{*}$, we might ignore the influence of $T$ on the terms $\mu_{2} \mathbf{z}_{k} \mathbf{H}^{-1} \mathbf{g}$, for $k \in\{1,2\}$, in (32). Then the minimum of $\gamma(T)$ would be given by

$$
\tilde{T}^{*}=\mu_{1} \sqrt{\frac{\left(s_{1}+s_{2}\right) 2 \rho_{1}\left(1-\rho_{1}\right)}{c_{1} \mu_{1}-c_{2} \mu_{2}}},
$$

which coincides in the limit $\rho \rightarrow 1$ with the diffusion approximation result obtained heuristically by Reiman and Wein [20], who assume that classes 1 and 2 are identified as high- and low-priority classes, respectively (i.e., $c_{1} \mu_{1} \geq c_{2} \mu_{2}$ ).

In the whole range of $\rho$, it becomes pretty difficult to prove that $\mu_{2} \mathbf{z}_{k} \mathbf{H}^{-1} \mathbf{g}$, for $k \in\{1,2\}$, do not affect the choice of the optimal threshold. But, in the limit $\rho \rightarrow 1$, an interesting implication of [20] is that it seems quite likely that the optimal choice of $T^{*}$ does not depend on them. We therefore surmise that a good choice of $T^{*}$ - close to the optimal threshold as $\rho$ tends to one - may be 1 if $\left[\tilde{T}^{*}\right]=0$, where [.] denotes integer part; otherwise, we may let $T^{*}$ be $\left[\tilde{T}^{*}\right]$ if $f\left(\left[\tilde{T}^{*}\right]\right) \leq f\left(\left[\tilde{T}^{*}\right]+1\right)$, and $\left[\tilde{T}^{*}\right]+1$ if $f\left([\tilde{T}]^{*}\right)>f\left(\left[\tilde{T}^{*}\right]+1\right)$

\section{Acknowledgements}

The authors wish to thank the anonymous referee and the associate editor for their helpful comments. The research of the second author was supported by the DGINV through project BFM2002-02189.

\section{References}

[1] P.S. Ansell, K.D. Glazebrook and I. Mitrani Threshold policies for a single-server queuing network, Probability in the Engineering and Informational Sciences 15 (2001), 15-33.

[2] S. Bhulai and G. Koole. On the structure of value functions for threshold policies in queueing models, Journal of Applied Probability 40 (2003), 613-622.

[3] S. Bhulai and F.M. Spieksma. On the uniqueness of solutions to the Poisson equations for average cost Markon chains with unbounded cost functions, Mathematical Methods of Operations Research 58 (2003), 221-236.

[4] O.J. Boxma, G.M. Koole and I. Mitrani. Polling models with threshold switching. In: Quantitative
Methods in Parallel Systems (Eds. F. Baccelli, A. Jean-Marie and I. Mitrani), pp. 129-140, SpringerVerlag, 1995.

[5] C. Buyukkoc, P. Varaiya and J. Walrand. The $c \mu$ rule revisited, Advances in Applied Probability $\mathbf{1 7}$ (1985), 237-238.

[6] E. Çinlar. Introduction to Stochastic Processes, Prentice-Hall, Inc., Englewood Cliffs, NJ, 1975.

[7] Q. Deng. A two-queue polling model with regularly varying service and/or switchover times. Stochastic Models 19 (2003), 507-526.

[8] R. Groenevelt, G. Koole and P. Nain. On the bias vector of a two-class preemptive priority queue, Mathematical Methods of Operations Research $\mathbf{5 5}$ (2002), 107-120.

[9] M. Hofri and K.W. Ross. On the optimal control of two queues with server setup times and its analysis, SIAM Journal on Computing 16 (1987), 399-420.

[10] S.M.R. Iravani and B. Kolfal. When does the $c \mu$ rule apply to finite-population queueing systems?, Operations Research Letters 31 (2005), 301-304.

[11] C. Knessl, D.I. Choi and C. Tier. A dynamic priority queue model for simultaneous service of two traffic types, SIAM Journal on Applied Mathematics 63 (2002), 398-422.

[12] G. Koole. Assigning a single server to inhomogeneous queues with switching costs, CWI Report BSR9405 (1994), 14 pages.

[13] G. Koole and P. Nain. On the value function of a priority queue with an application to a controlled polling model, Queueing Systems 34 (2000), 199-214.

[14] G. Koole and P. Nain. An explicit solution for the value function of a priority queue, Queueing Systems 47 (2004), 251-282.

[15] D.-S. Lee and B. Sengupta. Queueing analysis of a threshold based priority scheme for ATM networks, IEEE/ACM Transactions on Networking 1 (1993), 709-717.

[16] Z. Liu, P. Nain and D. Towsley. On optimal polling policies, Queueing Systems 11 (1992), 59-83.

[17] T.J. Ott and K.R. Krishnan. Separable routing: a scheme for state-dependent routing of circuit telephone traffic, Annals of Operations Research $\mathbf{3 5}$ (1992), 43-68.

[18] T. Prieto-Rumeau and O. Hernández-Lerma. The Laurent series, sensitive discount and Blackwell optimality for continuous-time controlled Markov chains, Mathematical Methods of Operations Research 61 (2005), 123-145. 
[19] M.L. Puterman. Markov Decision Processes: Discrete Stochastic Dynamic Programming, John Wiley \& Sons, Inc., New York, 1994.

[20] M.I. Reiman and L.M. Wein. Dynamic scheduling of a two-class queue with setups, Operations Research 46 (1998), 532-547.
[21] L. Takács. Introduction to the Theory of Queues, Oxford University Press, New York, 1962.

[22] L. Takács. Two queues attended by a single server, Operations Research 16 (1968), 639-650.

[23] J.A. Van Mieghem. Dynamic scheduling with convex delay costs: the generalized $c \mu$ rule, Annals of Applied Probability 5 (1995), 808-833. 